\title{
Genetically Engineered Indica Rice for Drought and Salinity Tolerance and Weed Management
}

\author{
Aakrati Agarwal ${ }^{1,2}$, Dhirendra Fartyal ${ }^{2}$, Yashwanti Mudgil ${ }^{1}$, Malireddy K. Reddy ${ }^{2}$ \\ ${ }^{1}$ Plant Molecular Biology Lab, Department of Botany, University of Delhi \\ Delhi-110007, India \\ aakrati@icgeb.res.in; dhirenfartyal@gmail.com \\ ${ }^{2}$ Crop Improvement Group, ICGEB, Aruna Asaf Ali Marg \\ New Delhi-110067, India \\ ymudgil@gmail.com; reddy@icgeb.res.in
}

\section{Extended Abstract}

By the year 2050, the geometric progression in population along with a drastic climate change will put an immense pressure on agricultural systems to feed 9 billion people globally [1]. Rice is a staple cereal to more than $50 \%$ of the world population and is highly sensitive to environmental stress [2]. Biotechnological tools aid in introducing cloned genes to provide abiotic stress tolerance and these traits are heritable $[3,4]$. In the present study, we aimed to analyse an altogether different strategy to solve the drought and salinity problem together. Therefore, we chose two genes from Pennisetum glaucum, LEA2, a dehydrin, commonly reported to provide drought tolerance and eIF4A2, a eukaryotic translation initiation factor, with a reported role in osmotic stress tolerance in animals and plants alike. A third gene bar from Streptomyces sps. was chosen to provide an additional character of herbicide tolerance. Gene pyramiding was done with these three genes and the transgenic rice plants were analyzed for the combined effects of $L E A 2$ and $e I F 4 A 2$ genes on drought and salt tolerance and the effect of bar gene on phosphinothricin tolerance in these transgenic rice plants. The objectives of the study included an in-depth genome-wide analyses of the roles of the above two selected genes, LEA2 and eIF4A2 in abiotic stress in rice; the cloning of the three genes with their respective promoters in a highly virulent Agrobacterium strain and transforming the gene construct into a responsive indica rice variety to produce rice transgenic lines. Further, the objectives were to analyze these transgenic rice plants for transient and then stable integration of genes, their expression via molecular analysis; the physiological analyses of these plants under simulated stress conditions and lastly to find the location of transgene integration in the rice genome. The scope of this study is to generate plants that can survive the combined stresses of salinity and drought better than the wild type plants due to over-expression of the two abiotic stress related genes. Further, the additional bar gene provides tolerance to the rice plants against the herbicide phosphinothricin which can be used to eliminate weeds from the rice field. This technology can be extended to the other abiotic stress sensitive indica rice varieties to combat climate change. The technology used in this study led to the generation of an adequate number of regenerants and transformants which were then further used for molecular analyses. Southern blotting analysis indicated a number of stable integrations in both single and multiple copies. Four transgenic lines were selected to measure their physiological performance under simulated drought and salinity stress conditions. Greenhouse experiments which checked the growth, biomass and photosynthetic parameters validated the effect of over-expression of the above genes in providing stress tolerance. Biochemical analyses showed better tolerance against stress in the transgenic lines. Semi-quantitative RT-PCR showed high expression of the three transgenes in the rice transgenic lines. Lastly, genome walking technique determined the position of the transgene in the chosen transgenic rice line.

\section{References}

[1] L. Lipper, N. McCarthy, D. Zilberman, S. Asfaw, and G. Branca, eds., Climate smart agriculture: building resilience to climate change. Springer International Publishing, 2018.

[2] S. H. Wani, and S. K. Sah, "Biotechnology and abiotic stress tolerance in rice," J. Rice Res., vol. 2, pp. 1-2, 2014. 
[3] P. S. Reddy, G. M. Reddy, P. Pandey, K. Chandrasekhar and M. K. Reddy, "Cloning and molecular characterization of a gene encoding late embryogenesis abundant protein from Pennisetum glaucum: protection against abiotic stresses," Mol. Biol. Rep., vol. 39, pp. 7163-7174, 2012.

[4] E. Chung, C. W. Cho, B. H. Yun, H. K. Choi, H. A. So, S. W. Lee and J. H. Lee, "Molecular cloning and characterization of the soybean DEAD-box RNA helicase gene induced by low temperature and high salinity stress," Gene., vol. 443, pp. 91-99, 2009. 